\title{
ORIGINAL ARTICLE \\ Sacral neuromodulation in patients with neurogenic lower urinary tract dysfunction
}

\author{
J Wöllner ${ }^{1}$, J Krebs ${ }^{2}$ and J Pannek ${ }^{1}$
}

Study design: This is a retrospective chart analysis.

Objectives: The objective of this study was to evaluate the effect of sacral neuromodulation (SNM) in patients with neurogenic lower urinary tract dysfunction (NLUTD).

Settings: This study was conducted in a spinal cord injury rehabilitation center in Switzerland.

Methods: The charts of all patients who underwent SNM (testing and/or permanent implantation) because of NLUTD at our institution between 2007 and 2013 were evaluated. Treatment outcomes and complications were recorded.

Results: A total of 50 patients, 30 women and 20 men, with a mean age of $46( \pm 14)$ years, fulfilled the inclusion criteria. The most frequent cause for SNM was spinal cord injury in 35 patients (70\%). Median duration of the underlying disease was 9.5 ( \pm 9.3 ) years. In all, 35 patients (70\%) received a permanent implant. The complication rate was 16\% (8/50). At the last follow-up, SNM was in use in 32 patients. In 26 patients with SNM because of detrusor overactivity, voiding frequency per 24 h was significantly reduced from 9 to 6 , and daily pad use rate was significantly improved (2.6 versus 0.6 pads per $24 \mathrm{~h}$ ). On comparing urodynamic assessment of detrusor function before and under SNM, no significant suppression of neurogenic detrusor overactivity (NDO) was detected. In nine patients with chronic neurogenic urinary retention, median postvoid residual urine was significantly reduced from 370 to $59 \mathrm{ml}$. In all, 94\% of the patients were either very satisfied or satisfied with SNM.

Conclusion: SNM might be an additional therapy option in carefully selected patients with NLUTD. On the basis of our results, urodynamic evaluation before SNM is mandatory, as the procedure does not seem to be suited to significantly alleviate NDO.

Spinal Cord (2016) 54, 137-140; doi:10.1038/sc.2015.124; published online 28 July 2015

\section{INTRODUCTION}

The physiological function of the lower urinary tract (LUT) is storage and controlled evacuation of urine. The LUT is controlled by spinal, supraspinal and cerebral networks. ${ }^{1-3}$ Because of the complexity of the neural interactions, damage of at least one of the components can cause LUT dysfunction (NLUTD). ${ }^{4}$ Depending on the neurologic disease and the location of the lesion, various forms of NLUTD can occur. Depending on the type of dysfunction, NLUTD can affect the upper urinary tract with the risk of renal failure, and it can impair the quality of life.,6 Therefore, treatment of NLUTD is not based on symptoms alone, but it should focus on the results of urodynamic testing.

Sacral neuromodulation (SNM) is a minimally invasive approach for the treatment of LUT dysfunction. The mechanism of action is not completely clarified, but a central modulation of afferent and efferent signals in the spinal cord and the supraspinal areas seems to have a crucial role. ${ }^{2}$ Although the efficacy and safety of SNM in patients with idiopathic LUT dysfunction has been frequently demonstrated, its effectiveness in patients with NLUTD is not well documented. ${ }^{7-10}$ In particular, there is a lack of clinical studies reporting urodynamic results after SNM.

The aim of this study was therefore to evaluate our experience with SNM in patients with NLUTD focusing on urodynamic results.

\section{MATERIALS AND METHODS \\ Patients}

In a retrospective chart analysis, we evaluated all patients receiving SNM for NLUTD in our institution between January 2007 and December 2013. The inclusion criterion was LUTD documented by urodynamic examination because of a neurologic disease/disorder. The data concerning demographics, the underlying disease, its duration and extent and previous therapies were collected.

\section{Sacral neuromodulation}

The interventions were performed by two experienced surgeons using bilateral tined leads implanted in the $\mathrm{S} 3$ sacral foramina. In case of inadequate response at the S3 level, S4 was chosen. The details of the implantation procedures are listed in Table 1. The standard modulation parameters during the test phase were bipolar modulation, pulse width $210 \mathrm{us}, 10-14 \mathrm{~Hz}$ and $1.5-10 \mathrm{~mA}$, depending on the patients' sensory threshold, with $10 \mathrm{~mA}$ as the upper limit for patients with absent or severely limited sensation. The criteria for successful test stimulation leading to permanent implantation were a symptom improvement of $>50 \%$ and patient satisfaction with the clinical result. ${ }^{11}$ The peri- and postinterventional complications were classified according to the Clavien-Dindo classification. ${ }^{12}$

\section{Follow-up examination}

Follow-up examination was performed 6 months after definite SNM implantation. It comprised assessment of bladder management by bladder diaries that

\footnotetext{
${ }^{1}$ Neuro-Urology, Swiss Paraplegic Center, Nottwil, Switzerland and ${ }^{2}$ Clinical Trial Unit, Swiss Paraplegic Center, Nottwil, Switzerland Correspondence: Professor J Pannek, Neuro-Urology, Swiss Paraplegic Center, Guido A Zäch Strasse 1, Nottwil 6207 Switzerland. E-mail: juergen.pannek@paraplegie.ch

Received 14 December 2014; revised 8 May 2015; accepted 23 June 2015; published online 28 July 2015
} 
Table 1 Implantation side and localization

\begin{tabular}{lr}
\hline Implantation site & $\mathrm{n}$ \\
\hline S3 bilateral & 35 \\
S4 bilateral & 1 \\
S3 and S4 & 1 \\
S3 unilateral & 4 \\
Neuralgia pudendus bilateral & 1 \\
S3 unilateral and N. pudendus unilateral & 4 \\
S4 unilateral & 1 \\
S3 right and S4 left & 3 \\
\hline
\end{tabular}

were administered 1 week before the control visit, urodynamic testing and evaluation of patient satisfaction. The urodynamic measurements and evaluations were performed according to the current International Continence Society standards. ${ }^{13}$ Patient satisfaction was evaluated with the 'Quality of Life Due to Urinary Symptoms' part of the American Urological Association Symptom Score 6 months after surgery, which has already been used in patients with nonobstructive and neurogenic LUT dysfunctions (neue Lit).

The urodynamic data recorded during the follow-up were compared with the urodynamic results before SNM testing. As a low compliance and elevated detrusor pressures during the storage phase are risk factors for renal impairment, we analyzed the effect of SNM on these parameters as primary end points.

\section{Statistical analyses}

Statistical analyses were performed with the SPSS statistical software (SPSS, Irvine, CA, USA). Because of normal distribution of the values (KolmogorowSmirnow test), paired and unpaired $t$-tests were used. All values were given as means and s.d. A $P$-value of $<0.05$ was considered significant.

\section{Statement of ethics}

This study was approved by the local ethics committee.

\section{RESULTS}

Between January 2007 and December 2013, 30 female and 20 male patients with a mean age of $46( \pm 14)$ years were evaluated. The mean duration of the underlying neurological disorder was $9.5( \pm 9.3)$ years. Incomplete spinal cord injury (SCI), present in $35 / 50$ patients $(70 \%)$, was the most common disorder. The other underlying neurological disorders are listed in Table 2.

One-third of the patients were ambulatory, and the remaining were either ambulatory with support or wheel chair bound (Table 2). The majority of the patients $(>70 \%)$ had received at least two previous NLUTD therapies.

\section{First-stage SNM (test phase)}

All 50 patients underwent a test phase. Implantation sites are listed in Table 1. The duration of the test phase was 21.3 ( \pm 11.2$)$ days. In 35 patients, a permanent impulse generator (IPG) (Interstim II or Interstim twin; Medtronic, Fridley, MN, USA) was implanted because of a positive test phase, whereas in 15 patients the electrodes were explanted because of a lack of efficacy. Test phase was positive in 26/39 (67\%) patients with neurogenic detrusor overactivity (NDO), and in 9/11 (82\%) individuals with urinary retention, respectively.

\section{Second-stage SNM (permanent implant)}

During the implantation of the 35 IPGs implanted because of a positive test phase, no adverse events were observed. We observed eight complications (Clavien-Dindo Classification ${ }^{14}$ grade IIIb): two
Table 2 Distribution of patient characteristics (age, gender), underlying disease, lesion level and duration of disease

\begin{tabular}{lc}
\hline Female & 30 \\
Male & 20 \\
Age & $46.3 \pm 14.2$ years \\
Duration of injury & $9.5 \pm 9.3$ years \\
Follow-up & $1.3 \pm 1.2$ years \\
Spinal cord injury & $35 / 50(705)$ \\
Myelomeningocele & $2 / 50(4 \%)$ \\
Multiple sclerosis & $2 / 50(4 \%)$ \\
Morbus Parkinson & $1 / 50(2 \%)$ \\
Other neurological disease & $10 / 50(20 \%)$ \\
& \\
AIS score (patients with SCI) & $\mathrm{n}=35$ \\
AIS A & 3 \\
AIS B & 2 \\
AIS C & 11 \\
AIS D & 19 \\
Lesion level & $\mathrm{n}=39$ \\
Cervical & 16 \\
Thoracic & $16(32 \%)$ \\
Lumbar & $18(36 \%)$ \\
Sacral & $16(32 \%)$ \\
Mobility & 12 \\
Ambulatory & 4 \\
Wheelchair & \\
\hline
\end{tabular}

Abbreviations: AIS, Abbreviated Injury Score; SCI, spinal cord injury.

explanations of the device because of infection, and the remaining six were technical defects of the devices.

At the last follow-up, $1.3( \pm 1.2)$ years after implantation, 32 IPGs were still in use, one IPG had been explanted because of infection (20 days after implantation), one was defective and one had been turned off because of a spillover of the modulation to nontarget systems (left lower extremity), ${ }^{15}$ which could not be managed by reduction of modulation intensity.

\section{Patients with NDO}

Thirty-nine patients suffered from NDO (23 females, 16 males), 28 of whom were incontinent. In 24 patients, a combination of NDO and detrusor sphincter dyssnergia (DSD)) was present. In $26(66.7 \%)$ of the NDO patients, the test phase was successful; 16 of these patients suffered from NDO and DSD.

At follow-up, 17 patients did not need additional treatment for NDO. Seven patients required additional antimuscarinic treatment, whereas two patients were on $\alpha$-blockers. Under SNM, 21 patients (80.8\%) reported complete continence, and five patients (19.2\%) were incontinent. Both the frequency of bladder drainage (e.g., spontaneous, intermittent catheterization) and of the pad use per $24 \mathrm{~h}$ were significantly reduced (Table 3 ).

Urodynamic investigations both before and after implantation were available in 15 of 26 patients. The mean time between postimplantation urodynamics and SNM implantation was 6.6 months. We observed a slight increase in the bladder capacity up to $262 \mathrm{ml}$, and an insignificant decrease in maximum detrusor pressure during the storage phase. The compliance increased significantly (Table 3). In 10 patients with NDO and DSD, no DSD could be detected under SNM in seven patients. Apart from the DSD, the urodynamic parameters 
Table 3 Results in patients with NDO and SNM treatment ${ }^{\mathrm{a}}$

\begin{tabular}{|c|c|c|c|c|}
\hline & $\mathrm{N}$ & Pre: mean \pm s.d. & Post: mean \pm s.d. & P-value \\
\hline \multicolumn{5}{|l|}{ Results SNM in NOAB patients } \\
\hline Frequency of bladder drainage per $24 \mathrm{~h}$ & 25 & $9.9 \pm 4.2$ & $5.7 \pm 1.5$ & $P<0.05$ \\
\hline Maximum bladder capacity (ml) & 15 & $246 \pm 112.7$ & $262 \pm 101.7$ & $P=0.59$ \\
\hline Pdet maximum in $\mathrm{cm} \mathrm{H}_{2} \mathrm{O}$ & 15 & $43.7 \pm 18.4$ & $38.4 \pm 18.4$ & $P=0.42$ \\
\hline Compliance $\left(\mathrm{ml} \mathrm{cmH}_{2} \mathrm{O}^{-1}\right)$ & 15 & $22.8 \pm 17.2$ & $53.6 \pm 55.8$ & $P<0.046$ \\
\hline
\end{tabular}

NDO, neurogenic detrusor overactivity; NOAB, neurogenic overactive bladder; Pdet maximum, maximum detrusor pressure during storage phase; SNM, sacral neuromodulation.

${ }^{a}$ By comparing the urodynamic results before the SNM test phase and the urodynamic results at the last follow-up.

did not differ significantly between the patients with NDO alone and those with NDO/DSD or between those with sufficiently treated DSD compared with those with persistent DSD, respectively.

\section{Patients with chronic neurogenic urinary retention}

Chronic neurogenic nonobstructive urinary retention was the indication for SNM in 11 patients (seven female and four male). Mean catheterization frequency of the patients performing intermittent catheterization was 5.5 per $24 \mathrm{~h}$. Nine out of eleven patients $(82 \%)$ had a positive test phase and received a permanent IPG. Before SNM treatment, only three patients were able to void without a catheter (one each spontaneous, abdominal strain, reflex micturition). Three patients had suffered from incontinence because of NDO. After implantation, all nine were able to void spontaneously, and all patients achieved continence. SNM treatment led to a significant reduction of the postvoid residual urine from 370 to $59 \mathrm{ml}$. In addition, pad use was slightly reduced, whereas the frequency of bladder evacuation remained unchanged. With regard to the urodynamic parameters, maximum bladder capacity, detrusor pressure and compliance did not change significantly under SNM treatment.

\section{Patient satisfaction}

Of the 32 patients with SNM in use at the last follow-up, 21 were very satisfied, 9 were satisfied, 1 was unchanged and 1 was unsatisfied.

\section{DISCUSSION}

SNM is a well-established treatment option for patients with idiopathic lower urinary tract signs. A systematic review from 2010, however, came to the conclusion that the number of investigated patients with SNM for the treatment of NLUTD was low, with high between-study heterogeneity, and that there was a lack of randomized, controlled trials. ${ }^{10}$ In the absence of prospective studies, welldocumented case series are useful to increase our knowledge about the usefulness of SNM in patients with NLUTD. According to our knowledge, we present the largest single-center experience of SNM in this group of patients. Our results demonstrate that SNM is a safe and effective procedure in carefully selected symptomatic patients with NLUTD.

On the other hand, as NDO and a low compliance are the most important risk factors for renal damage in patients with NLUTD, ${ }^{16}$ treatment should be based not merely on symptoms alone but also on urodynamic assessment of risk factors for renal impairment. Only a few previous retrospective case series with small numbers of participants evaluated the urodynamic outcomes of SNM in NLUTD. Lombardi and Del Popolo ${ }^{17}$ presented the data from 24 patients with NLUTD because of incomplete SCI: 13 patients with chronic retention and 11 patients with NDO. In their study, 5 of the 13 patients with chronic retention did not require intermittent catheterization (IC) anymore. In the patients with NDO combined with DSD, neither objective nor subjective SNM success was observed, whereas in patients with pure NDO, a significant decrease in incontinence and in urodynamic parameters could be observed. ${ }^{17}$ Chaabane et al. ${ }^{18}$ presented a retrospective case series with 62 patients suffering from NLUTD: the majority from multiple sclerosis (MS) or incomplete SCI (13 patients each) and the remaining patients from various neurologic disorders. In their study, SNM led to a significant improvement not only of symptoms but also of urodynamic parameters including improvement of DSD in eight of nine patients.

Comparing the urodynamic results from the mentioned studies with our results, some differences are striking. Whereas we did not observe any significant improvement in NDO during the storage phase, NDO was successfully treated in the other two studies. Furthermore, the results of the two mentioned studies are contradictory. Whereas Lombardi did not find any improvement if NDO was combined with DSD, Chaabane et al. ${ }^{18}$ treated eight of nine patients with DSD successfully. One of the series, however, is severely limited by the small sample size, whereas in the other publication only a minority of the patients suffered from SCI or MS, the two disorders that were present in the majority of our patients. In addition, exclusively SCI patients with less complete lesions (ASI C and D) were included in the mentioned studies, whereas AIS B patients were included in our patient cohort. In our series, DSD did not influence subjective or objective outcome, although it was not detectable any more in 7 of 10 patients with the initial diagnosis of DSD.

Previous publications conclude that SNM should be considered in incomplete SCI patients with NDO or chronic retention, as SNM is effective in both forms of NLUTD. ${ }^{18}$ On the basis of our results, however, SNM cannot reliably suppress NDO. An elevated detrusor pressure during the storage phase can affect the function of the upper urinary tract, possibly leading to renal failure. ${ }^{19}$ Elevated detrusor pressures are not associated with the patients' symptoms. ${ }^{20,21}$ Therefore, we do not offer SNM routinely to each patient who cannot be successfully treated conservatively.

As in all SCI patients with NLUTD ${ }^{18}$ regular urodynamic follow-up is important also after implantation of SNM. Seven SCI patients with successful SNM treatment of NDO in the test phase required additional antimuscarinic treatment during follow-up. Our findings are in concordance with other studies reporting a loss of SNM efficacy in four patients in whom a worsening of the neurological status and technical problems could be ruled out. ${ }^{17}$ Loss of SNM function seems to occur even more frequently in MS patients. Chaabane et al. demonstrated a loss of SNM effect in $41 \%$ of the MS patients, most often because of progressive neurological disease. As especially MS patients require frequent MRI controls, a diagnostic procedure that is discussed as being incompatible with SNM implantation, especially MS patients should be carefully selected for this procedure. ${ }^{8}$ 
In summary, according to our findings, SNM seems to be a treatment option in carefully selected patients with symptomatic NLUTD without a high risk for upper urinary tract damage. Regular urodynamic evaluation before SNM and after implantation is mandatory.

The limited number of patients and the retrospective nature, however, pertain to the limitations of our study. Although urodynamic follow-up is usually performed in every patient with NLUTD after a surgical procedure, we were not able to present urodynamic data in all patients after SNM implantation, which is one important drawback of our study. As we serve as a referral center for the majority of SCI patients in our country, however, a significant number of patients did not return to our department, but were managed by their local urologists. Nonetheless, this study presents the largest experience with urodynamic results after SNM in patients with NLUTD, and therefore the results are of substantial interest. In addition, the lack of data and the important consequences for patient selection and management if our findings can be confirmed underline the need for a prospective evaluation of the urodynamic effects of SNM on NLUTD.

\section{DATA ARCHIVING}

There were no data to deposit.

\section{CONFLICT OF INTEREST}

The authors declare no conflict of interest.

1 Holstege G, Mouton LJ. Central nervous system control of micturition. Int Rev Neurobiol 2003; 56: 123-145.

2 Fowler CJ, Griffiths D, de Groat WC. The neural control of micturition. Nat Rev Neurosci 2008; 9: 453-466.

3 Drake MJ, Fowler CJ, Griffiths D, Mayer E, Paton JF, Birder L. Neural control of the lower urinary and gastrointestinal tracts: supraspinal CNS mechanisms. Neurourol Urodyn 2010; 29: 119-127.

4 Panicker JN, Menon L, Anandkumar A, Sundaram KR, Fowler CJ. Lower urinary tract symptoms following neurological illness may be influenced by multiple factors: observations from a neurorehabilitation service in a developing country. Neurourol Urodyn 2010; 29: 378-381.

5 Akkoç Y, Ersöz M, Yıldız N, Erhan B, Alaca R, Gök H et al. Effects of different bladder management methods on the quality of life in patients with traumatic spinal cord injury. Spinal Cord 2013; 51: 226-231.

6 Bruschini H, Almeida FG, Srougi M. Upper and lower urinary tract evaluation of 104 patients with myelomeningocele without adequate urological management. World J Urol 2006; 24: 224-228.

7 Peters KM, Kandagatla P, Killinger KA, Wolfert C, Boura JA. Clinical outcomes of sacral neuromodulation in patients with neurologic conditions. Urology 2013; 81: 738-743.

8 Lay AH, Das AK. The role of neuromodulation in patients with neurogenic overactive bladder. Curr Urol Rep 2012; 13: 343-347.

9 Sievert KD, Amend B, Gakis G, Toomey P, Badke A, Kaps HP et al. Early sacral neuromodulation prevents urinary incontinence after complete spinal cord injury. Ann Neurol 2010; 67: 74-84.

10 Kessler TM, La Framboise D, Trelle S, Fowler CJ, Kiss G, Pannek J et al. Sacral neuromodulation for neurogenic lower urinary tract dysfunction: systematic review and meta-analysis. Eur Urol 2010; 58: 865-874.

11 Amend B, Khalil M, Kessler TM, Sievert KD. How does sacral modulation work best? Placement and programming techniques to maximize efficacy. Curr Urol Rep 2011; 12: 327-335.

12 Dindo D, Demartines N, Clavien PA. Classification of surgical complications: a new proposal with evaluation in a cohort of 6336 patients and results of a survey. Ann Surg 2004; 240: 205-213.

13 Schafer W, Abrams P, Liao L, Mattiasson A, Pesce F, Spangberg A et al. Good urodynamic practices: uroflowmetry, filling cystometry, and pressure- flow studies. Neurourol Urodyn 2002; 21: 261-274.

14 Clavien PA, Barkun J, de Oliveira ML, Vauthey JN, Dindo D, Schulick RD et al. The Clavien-Dindo classification of surgical complications: five-year experience. Ann Surg 2009; 250: 187-196.

15 Schiefer MA, Freeberg M, Pinault GJ, Anderson J, Hoyen H, Tyler DJ et al. Selective activation of the human tibial and common peroneal nerves with a flat interface nerve electrode. J Neural Eng 2013; 10: 056006.

16 Gerridzen RG, Thijssen AM, Dehoux E. Risk factors for upper tract deterioration in chronic spinal cord injury patients. J Urol 1992; 147: 416-418.

17 Lombardi G, Del Popolo G. Clinical outcome of sacral neuromodulation in incomplete spinal cord injured patients suffering from neurogenic lower urinary tract symptoms. Spinal Cord 2009; 47: 486-491.

18 Chaabane W, Guillotreau J, Castel-Lacanal E, Abu-Anz S, De Boissezon X, Malavaud B et al. Sacral neuromodulation for treating neurogenic bladder dysfunction: clinical and urodynamic study. Neurourol Urodyn 2011; 30: 547-550.

19 Stohrer M, Blok B, Castro-Diaz D, Chartier-Kastler E, Del Popolo G, Kramer G et al. EAU guidelines on neurogenic lower urinary tract dysfunction. Eur Urol 2009; 56: 81-88.

20 Nosseir M, Hinkel A, Pannek J. Clinical usefulness of urodynamic assessment for maintenance of bladder function in patients with spinal cord injury. Neurourol Urodyn 2007; 26: 228-233.

21 Gottsch HP, Miller JL, Yang CC, Berger RE. A pilot study of botulinum toxin for interstitial cystitis/painful bladder syndrome. Neurourol Urodyn 2011; 30: 93-6. 